\title{
Anisakiasis no es problema de salud pública
}

\author{
Anisakiasis is not a public health problem
}

A raíz de la noticia periodística, sobre la presencia del parásito anisakis hallado en un lote de conserva de caballa, cuyo fabricante es la empresa China TFM, se originó una alarma en Perú, generando miedo, confusión y malestar para no consumir cualquier tipo de conservas de pescado. Es por ello que el presente editorial busca presentar la situación de este parásito en nuestro país mediante una breve revisión actualizada del tema.

En las últimas dos décadas, el Perú, debido a su gran biodiversidad ha logrado que su gastronomía crezca y se desarrolle de manera importante a nivel nacional y mundial, destacando uno de sus platos favoritos, el ceviche peruano, el cual es de gran consumo popular; preparado a base de pescado crudo donde se agregan limón, cebolla, entre otros ingredientes. Felizmente en este plato bandera peruano como en otros a base de pescado fresco, no existe la enfermedad de anisakiasis y por lo tanto no es un problema de salud pública; en cambio, en Perú, se han reportado algunos casos de otra parasitosis poco conocida: gnatostomiasis, que es una zoonosis del nematodo del género gnathostoma, que incluye a diversas especies (1); fue descrito por primera vez en 1836, en un tumor de estómago de un tigre muerto en Londres (2), en 1989 se describió el primer caso humano y en América, las primeras descripciones se publicaron en México en la década de 1970. Posteriormente fueron apareciendo casos aislados en todo el mundo; esta enfermedad es endémica en el sudeste de Asia, India, Tailandia, Malasia, China y Japón, también en Australia, África y Europa. En América, se ha encontrado en México, Guatemala, Brasil, Ecuador y Perú $(2-9)$.

En nuestro país la gnatostomiasis fue descrita en 1987 en un paciente de consulta particular, posteriormente once casos fueron descritos el 2011 (2,3), siendo el grupo más afectado, la clase social alta, donde destacó el consumo de pescado crudo en forma de ceviche o comida japonesa. De las formas clínicas destaca por lejos la forma cutánea, y en raras ocasiones la ocular y visceral $(2,6,7,9)$.

La anisakiasis es otra zoonosis, causada por un nemátodo del género anisakis; los delfines, lobos marinos, ballenas y focas son los huéspedes naturales, de allí, el anisakis puede ingresar a pulpos, calamares y algunos peces como salmón, bacalao, caballa, etc., que al ser consumidos de manera cruda o semicruda, pueden causar en las personas, un daño agudo a nivel intestinal o causar alergias. Es de alta prevalencia en el Asia, especialmente en el Japón, donde se ha estimado una tasa de 2000 casos por año; en algunos países de Europa es de 500 casos por año (10). Con el devenir de los años se han ido reportando más casos en muchas partes del mundo, especialmente en Asia, Europa y Estados Unidos, probablemente asociado al consumo de pescado fresco en preparaciones tipo sushi y sashimi (11-13); en España, se lo ha asociado a la ingesta de boquerón en vinagre $(11,14)$. En Latinoamérica, en México el cebiche sería la fuente de infección, en Chile el incremento de los casos se debería al consumo de cebiche, sashimi y pescado ahumado.

En Chile, en 1980, se comunicaron casos de infección humana producida por Pseudoterranova sp (15-17). En nuestro país se han reportado dos casos por $P$. decipiens en Lima, en personas que habían ingerido "cebiche" (18); otro reporte de anisakiasis gástrica por larvas de Anisakis sp., en una mujer con antecedentes de ingesta de "cebiche" (19), y recientemente, dos casos de localización gástrica por larvas de A. physeteris en la ciudad de Ica, por ingesta de "cebiche" de Coryphaenahippurus; este hallazgo probablemente fue asociado al fenómeno El Niño de 1997-98 (20).

En total, en Perú, se han reportado ocho casos humanos de los cuales cinco son comprobados, tres de ellos producidos por larvas de P. decipiens (12), uno por larva de Anisakis sp., y otro por A. simplex; estos casos aislados 
a nivel gástrico e intestinal, presentaron dolor abdominal severo que requirió tratamiento sintomático, felizmente sin causar la muerte. Existen dos síndromes clásicos, el gastrointestinal y el alérgico, en la forma gastrointestinal, la anisakiasis puede ser gástrica $(70 \%)$, intestinal (20\%) o ectópica, si se encuentra fuera del tubo digestivo. Los síntomas pueden ser variados desde un dolor abdominal simple a un cuadro de abdomen agudo (apendicitis, ileitis). Uno de los autores encontró que hasta $60 \%$ de los casos fueron diagnosticados de apendicitis, cáncer gástrico o enfermedad de Crohn (21-23). Los cuadros asintomáticos generalmente son debidos a que el parásito no invade la mucosa (forma luminal), característico de las larvas de Pseudoterranova decipiens, y el diagnóstico en estos casos se realiza de forma casual al realizar una exploración endoscópica por otro motivo, identificando las larvas libres, o al ser expulsados en forma de vómitos o en las heces.

Las manifestaciones alérgicas sistémicas pueden variar desde la urticaria o angioedema hasta el shock anafiláctico, como cualquier otra reacción alérgica mediada por IgE (24). Lamentablemente, no hay terapia antiparasitaria para este parasito. Para evitar ser afectado, existen diversas medidas de prevención, como educar a los pescadores artesanales para que evisceren el pescado antes de someterlo a congelación para evitar que las larvas puedan migrar al músculo (11,12,25-27). Además, se recomienda que el pescado eviscerado debe estar congelado en altamar o ultracongelado, así la intensidad de infección muscular sería menor que el pescado eviscerado en la caleta o en el puerto. Asimismo, en lo posible se debe evitar consumir pescado crudo o insuficientemente cocinado de especies que contienen anisákidos patógenos. Puede comerse solo si es congelado a $-20^{\circ} \mathrm{C}$ por $48 \mathrm{~h}$ ó $72 \mathrm{~h}$ o cocinado por 10 minutos a más de $60^{\circ} \mathrm{C}$.

Finalmente consideramos que si bien se han reportado algunos escasos casos de anisakiasis en Perú, por las condiciones climáticas actuales ambientales y ecológicas, esta zoonosis no constituye un problema de salud pública, lo que se debe hacer es vigilar y estudiar la verdadera magnitud de este parasito en los diversos reservorios.

Ciro Maguiña Vargas 1,a;2, b

\section{REFERENCIAS BIBLIOGRÁFICAS}

1. Bravo E. Paniculitis migratoria eosinofílica en el Perú. Fol Dermatol. 2000; 2(2):7.

2. Costa AH, Bravo PFG, Valdez L, et al. Paniculitis nodular migratoria eosinofílica en el Perú. (Gnothostomiasis humana). Informe de once casos, posibles causas y revisión de la literatura. Fol Dermatol. 2001; 12(2):21-35.

3. Ollague W, Ollague J, Guevara de VelizA, Peñaherrera S. Human gnathostomiasis in Ecuador (nodular migratory eosinophilic panniculitis). First finding of the parasite in South America. Int J Dermatol. 1984; 23(10):647-51.

4. Almeyda-Artigas RJ, Bargues MD, Mas-Coma S. ITS-2 rDNA sequencing of Gnathostoma species (Nematoda) and elucidation of the species causing human gnathostomiasis in the Americas. J Parasitol. 2000; 86(3):537-44.

5. Lamothe-Argumedo R. La gnatostomiasis en Mexico: un problema de salud pública. Ann Inst Biol UNAM. 2003; 74:99-103.

6. Herman JS, Chiodini PL. Gnathostomiasis, another emerging imported disease. Clin Microbiol Rev. 2009; 22(3):484-92. doi: 10.1128/CMR.00003-09
7. Álvarez P, Morales A, Bravo F. Gnatostomiasis, experiencia en una práctica privada en Lima-Perú. Folia dermatol Peru. 2011; 22(2):67-74.

8. Laga AC, Lezcano C, Ramos C, et al. Cutaneous gnathostomiasis: report of 6 cases with emphasis on histopathological demonstration of the larva. J Am Acad Dermatol. 2013; 68(2):301-5. doi: 10.1016/j.jaad.2012.07.016

9. Villar E. Paniculitis migratoria eosinofílica en el Perú: Gnathostoma como agente causal. Rev perú med exp salud pública. 2003; 20(4):220-222.

10. Panel on Biological Hazards. Risk assessment on parasites in fishery products. EFSA Journal. 2010; 8(4):1543. doi: 10.2903/j.efsa.2010.1543

11. McCarty J, Moore T. Emerging helminth zoonoses. Int J Parasitol. 2000; 30:1351-1360.

12. Cabrera R, Trillo-Altamirano M. Anisakidosis: ¿Una zoonosis parasitaria marina desconocida o emergente en el Perú? Rev gastroenterol Perú. 2004; 24(4):335342.

13. Oldfield E. Emerging foodborne pathogens: keeping your patients and your families safe. Rev Gastroenterol Disord. 2001; 1:177-186.

14. Audicana MT, Ansotegui IJ, de Corres LF, Kennedy MW. Anisakis simplex: dangerous-dead 
and live? Trends Parasitol. 2002; 18:20-25.

15. Laffon-Leal SM, Vidal-Martínez VM, ArjonaTorres G. 'Cebiche' a potential source of human anisakiasis in Mexico? J Helminthol. 2000; 74:151154.

16. Mercado R, Torres P, Muñoz V, Apt W. Human infection by Pseudoterranova decipiens (Nematoda, Anisakidae) in Chile: report of seven cases. Mem Inst Oswaldo Cruz. 2001; 96(5):653-5.

17. Apt W, Hisamoto T, Llorens P, Alcaíno $H$. Anisakiasis gástrica en Chile. Rev Méd Chile. 1980; 108:825-827.

18. Tantalean VM, Huiza FA. Nematode larvae with medical importance found in sea fish from the Peruvian shore, with two records of human infections. Revista Peruana de Medicina Tropical. 1993; 7:61-65.

19. Barriga J, Salazar F, Barriga E. Anisakiasis: presentación de un caso y revisión de la literatura. Rev Gastroent Perú. 1999; 19:317-323.

20. Cabrera R, Suarez-Ognio L. Probable emergencia de anisakiosis por larvas de Anisakis physeteris durante el fenómeno El Niño 1997-98 en la costa peruana. Parasitol latinoam. 2002; 57(3-4):166170 .
21. Shimamura Y, Muwanwella N, Chandran S, Kandel G, Marcon N. Common Symptoms from an Uncommon Infection: Gastrointestinal Anisakiasis. Can J Gastroenterol Hepatol. 2016; 2016:5176502.

22. Repiso A, Alcantara M, Gonzalez de Frutos C. et al, Anisakiasis gastrointestinal (Estudio de una serie de 25 pacientes). Gastroenterol Hepatol. 2003; 26:341-346.

23. Sakanari JA, McKerrow JH. Anisakiasis. Clin Microbiol Rev. 1989; 2:278-284.

24. López D, Ramírez LM, del Rosal R, et al. Anisakiasis en España: una enfermedad creciente. Revisión. Gastroenterol Hepatol. 2000; 23:307-11.

25. Daschner A, Alonso-Gómez A, Cabañas R, Suárez de Parga M, López Serrano MC. Gastroallergic anisakiasis: Bordeline between food allergy and parasitic disease: Clinical and allergologic evaluation of 20 patients with confirmed acute parasitism by Anisakis simplex. J Allergy Clin Immunol. 2000; 105:176-81.

26. Chin J, editor. Manual para el control de las enfermedades transmisibles. $17 \mathrm{ma}$ ed. Washington DC: OPS/OMS; 2001.

27. Domínguez-Ortega J, Martínez-Cócera C. Guidelines in pathology induced by Anisakis simplex. Alergol Inmunol Clin. 2000; 15:267-272.

Facultad de Medicina Alberto Hurtado, Universidad Peruana Cayetano Heredia. Lima, Perú.

Departamento de Enfermedades Infecciosas Tropicales y Dermatológicas. Hospital Nacional Cayetano Heredia. Lima, Perú.

Docente; ${ }^{\mathrm{b}}$ Médico Infectólogo, Tropicalista, Dermatólogo. 